\title{
IOLOGIA COMPORTAMENTAL COMPARADA ENTRE MOLUSCOS TERRESTRES NATIVOS E EXÓTICOS
}

\section{Vinícius José Pilate}

Programa de Pós-graduação em Ecologia Aplicada ao Manejo e Conservação de Recursos Naturais, Universidade Federal de Juiz de Fora, R. José Lourenço Kelmer, s/no, Martelos, CEP: 36.036-330, Juiz de Fora, MG, Brasil. E-mail: viniciuspilate@gmail.com

\section{Evelyn Durço Chicarino}

Programa de Pós-graduação em Ciências Biológicas - Comportamento e Biologia Animal, Universidade Federal de Juiz de Fora, R. José Lourenço Kelmer, s/no, Martelos, CEP: 36.036-330, Juiz de Fora, MG, Brasil. E-mail: evelynbiobacharel@gmail.com

\section{Lidiane Cristina da Silva}

Programa de Pós-graduação em Ciências Veterinárias - Parasitologia Veterinária, Universidade Federal Rural do Rio de Janeiro, Rod. BR 465, Km 7, s/no, Zona Rural, CEP: 23.890-000, Seropédica, RJ, Brasil. E-mail: lidianecrissilva@gmail.com

\section{TÉrCia Vargas dos Santos}

Programa de Pós-graduação em Entomologia, Universidade Federal de Viçosa, Av. Peter Henry Rolfs, s/nº, Campus Universitário, CEP: 36.570-900, Viçosa, MG, Brasil.

E-mail: terciavargas@gmail.com

\section{Bruna Aparecida de Souza}

Programa de Pós-graduação em Química, Universidade Federal de Minas Gerais, Av. Presidente Antônio Carlos, 6.627, Pampulha, CEP: 31.270-901, Belo Horizonte, MG, Brasil. E-mail: brunasouzamk21@gmail.com

\section{Elisabeth Cristina de Almeida Bessa}

Departamento de Zoologia, Instituto de Ciências Biológicas, Universidade Federal de Juiz de Fora, R. José Lourenço Kelmer, s/no, Martelos, CEP: 36.036-330, Juiz de Fora, MG, Brasil. E-mail: elisabeth.bessa@ufjf.edu.br

\begin{abstract}
Resumo: Objetivou-se com este estudo verificar o horário de atividade, a frequência dos atos comportamentais através do etograma básico e a influência da temperatura e da umidade relativa do ar sobre o comportamento de Achatina fulica Bowdich, 1822 e Megalobulimus oblongus (Müller, 1774). Ambas as espécies apresentaram atividade noturna. Os atos comportamentais mais frequentes foram "repousar", "estar enterrado" e "alimentar" para as duas espécies. Megalobulimus oblongus foi menos ativo que a espécie exótica, porém apresentou frequência alimentar maior. O aumento da temperatura causou a redução da atividade de ambas as espécies, enquanto o aumento da umidade apenas influenciou a atividade de $A$. fulica. Apesar de a espécie nativa ter se mostrado menos suscetível às mudanças na temperatura e umidade, e ter se alimentado mais que a exótica, as características biológicas e ecológicas de $A$. fulica, como seu grande potencial reprodutivo e a ausência de predadores naturais, fazem desta um competidor mais eficiente, capaz de colocar as populações de M. oblongus em risco de extinção.
\end{abstract}

Palavras-chave: Atividade, Etograma, Gastrópodes, Temperatura, Umidade. 


\title{
BEHAVIORAL BIOLOGY COMPARED BETWEEN NATIVE AND EXOTIC TERRESTRIAL MOLLUSCS
}

\begin{abstract}
The aim of this study was to verify the activity period, the frequency of behavioral acts through basic ethogram and the influence of temperature and the relative air humidity on behavior of Achatina fulica Bowdich, 1822 and Megalobulimus oblongus (Müller, 1774). Both species presented nocturnal activity. The most frequent behavioral acts were "Rest", "Buried" and "Feed" for both species. Megalobulimus oblongus was less active than the alien species, but presented a higher feeding frequency. The increasing temperature caused reduction in activity on both species while the increase in humidity influenced only $A$. fulica. Despite the native species being less susceptible to changes in temperature and humidity and fed more than the alien species, the biological and ecological features of $A$. fulica, as its great reproductive potential and the absence of natural predators, make this species an efficient competitor, with potential to extinct the populations of M.oblongus.
\end{abstract}

Keywords: Activity, Ethogram, Gastropods, Temperature, Humidity.

\section{INTRODUÇÃO}

A introdução de espécies exóticas é considerada a segunda maior causa de extinção de espécies nativas, o que afeta diretamente a biodiversidade (Coradin \& Tortato, 2006). A espécie Achatina fulica Bowdich, 1822 (Achatinidae) é um molusco pulmonado terrestre nativo da região leste-nordeste da África (Vasconcellos \& Pile, 2001), atualmente considerado uma das cem piores espécies invasoras no mundo, devido às suas características biológicas, à grande resistência aos fatores ambientais e a hábitos alimentares generalistas (IUCN, 2016). Nas áreas onde foi introduzido, incluindo o Brasil, A. fulica se tornou um sério problema ambiental, em consequência da sobreposição de nicho (Eston et al., 2006) e da competição por alimento e locais de reprodução com a fauna nativa (Colley \& Fisher, 2009).

O impacto causado por espécies invasoras evidencia a ineficiência na prevenção, na detecção e no controle das invasões (Byers et al., 2002). O controle das populações de gastrópodes terrestres não é fácil, uma vez que, para sobreviver, eles exibem estratégias comportamentais, como retração da massa cefalopediosa e fuga, que inutilizam os procedimentos adotados (D'ávila et al., 2004; Storey, 2002). A ausência de controle permite que populações de $A$. fulica se alastrem rapidamente para áreas antropizadas e para ambientes naturais (Eston et al., 2006). Há indícios de que $A$. fulica esteja causando direta ou indiretamente a diminuição das populações de moluscos da família Megalobulimidae, principalmente Megalobulimus oblongus (Müller, 1774), molusco nativo do território brasileiro ( $\mathrm{Fi}-$ sher \& Colley, 2005).

As pesquisas sobre biologia comportamental de moluscos terrestres tendem a contribuir não só com medidas de controle de espécies invasoras, como é o caso da $A$. fulica, mas também com estratégias de conservação de espécies ameaçadas, como M. oblongus (D'ávila et al., 2004; Storey, 2002). Tais pesquisas têm diversas abordagens, tais como: etograma e horário de atividade (Chicarino et al., 2013; Grimm \& Schaumberger, 2002; Junqueira et al., 2003, 2004; Pilate et al., 2012, 2013b, 2015; Silva et al., 2011; Souza et al., 2013), agregação e preferência por sítio de repouso (Bohan et al., 2000; Chase, Croll \& Zeichner, 1980; D'ávila, Dias \& Bessa, 2006; Dundee, Tizzard \& Traub, 1975; Kleewein, 1999; Lazaridou-Dimitriadou \& Daguzan, 1981; Pilate et al., 2013a; Silva et al., 2011), estivação, forrageamento, comunicação e reprodução (Bailey, 1989; Chatfield, 1976; Chevalier et al., 2000; Cook, 1985, 1992; Emberton, 1994; Iglesias \& Castillejo, 1999; Leahy, 1983; Pakarinen, 1992; Raut \& Panigrahi, 1988, 1990; Stephenson 1979), e efeito de fatores bióticos e ambientais (Bailey, 1981; Hodasi, 1982; Junqueira et al., 2003).

O objetivo deste estudo foi verificar o horário de atividade de $A$. fulica e $M$. oblongus, a frequência dos atos comportamentais exibidos por ambas as espécies, através do etograma básico, e a influência da temperatura e da umidade relativa do ar na atividade destes animais.

\section{MATERIAL e MÉtodos}

Para a realização deste trabalho, foram utilizados 15 indivíduos adultos da espécie $A$. fulica e 15 da espécie $M$. oblongus, sendo estes obtidos de criações matrizes formadas a partir de moluscos reunidos na Câmara Municipal de Valença, Rio de Janeiro (22S 14'44", 43W 42'01"), e mantidas no Laboratório de Moluscos Arnaldo Campos dos Santos Coelho, do Museu de Malacologia Professor Maury Pinto de Oliveira,da Universidade Federal de Juiz de Fora, Minas Gerais, onde 
o experimento foi desenvolvido. Essa quantidade de animais foi utilizada em face da dificuldade de obter M. oblongus, que são raros e ocorrem em baixas densidades, de modo a evitar mais impactos em suas populações.

Os moluscos foram acondicionados em terrários de $34 \mathrm{~L}$ (três repetições por espécie de cinco moluscos cada), contendo $5 \mathrm{~kg}$ de terra vegetal comercial esterilizada ( $120^{\circ} \mathrm{C}$ durante $1 \mathrm{~h}$ ) e umedecida com água de torneira, e selados com pano de algodão e tiras de borracha. Os animais foram alimentados com uma mistura de ração para aves de corte e carbonato de cálcio (3:1) (Bessa \& Araújo, 1995a,b).

O estudo foi desenvolvido durante a fotofase (fase clara - período compreendido entre 6:00 h e 17:59 h) e a escotofase (fase escura - período compreendido entre 18:00 h de um dia e 5:59 h do dia seguinte), através do método "Scan Sample" (Altmann, 1974), com registro dos atos comportamentais descritos por Junqueira et al. (2004) e Pilate et al. (2012) a intervalos de 20 min, totalizando $24 \mathrm{~h}$ de observação. As observações noturnas foram realizadas usando uma lanterna embrulhada em papel celofane vermelho para proporcionar um comprimento de onda mais baixo, a fim de reduzir a interferência no comportamento dos animais.

O trabalho foi realizado no mês de abril sob condições naturais de temperatura e umidade relativa do ar, as quais foram registradas a cada observação através de um termômetro de máxima e de mínima e de um termo-higrômetro de bulbo seco e úmido, respectivamente.

A comparação entre a atividade, o repouso e a frequência dos atos comportamentais registrados para as duas espécies foi realizada por meio da análise de variância de Kruskal-Wallis, seguida pelo teste de Student-Newman-Keuls. Para verificar a influência da temperatura e da umidade relativa do ar nos atos comportamentais dos moluscos, utilizou-se o coeficiente de correlação de Spearman. As análises foram realizadas no programa BioEstat 5.0, adotando-se índice de significância de 0,05.

\section{Resultados}

Foram registrados nove atos comportamentais no total, sendo dois de repouso: "repousar" e "estar enterrado", e sete de atividade: "deslocar", "enterrar", "explorar", "alimentar", "defecar", "emergir" e "interagir", totalizando 1.097 registros. Os comportamentos de corte, cópula, oviposição, escavação de ninho e liberação de fiIhotes não foram registrados.

Para ambas as espécies, foi verificada maior atividade na escotofase $(H=190,86 ; p<0,01$ para A. fulica; e $\mathrm{H}=131,63 ; \mathrm{p}<0,01$ para $M$. oblongus), sendo que neste período a espécie $A$. fulica apresentou maior atividade do que $M$. oblongus $(\mathrm{H}=130,82 ; \mathrm{p}<0,01)$ (Fig. 1). Durante a fotofase, $A$. fulica também se apresentou mais ativa do que $M$. oblongus ( $\mathrm{H}=71,59 ; \mathrm{p}=0,04)$ (Fig. 1 ).

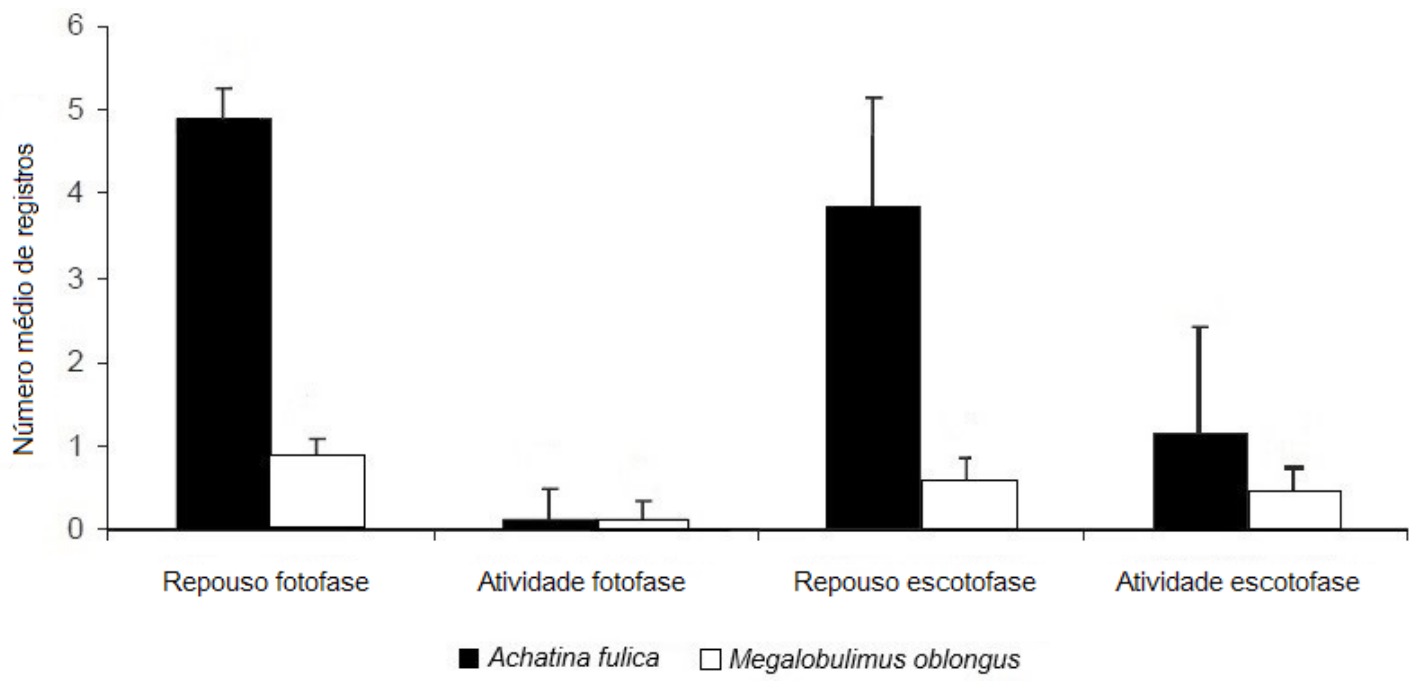

Fig. 1. Número médio de registros de atividade e repouso de Achatina fulica Bowdich, 1822 e Megalobulimus oblongus (Müller, 1774), durante a fotofase e a escotofase, em 24 horas consecutivas de observação em laboratório. 
Observou-se que os atos comportamentais "estar enterrado" (65\%), "repousar" (21\%) e "alimentar" ( $5 \%)$ foram os mais frequentes para A. fulica. O mesmo foi observado para M. oblongus, sendo as frequências de "estar enterrado", "repousar" e "alimentar" iguais a 30\%, 29\% e $21 \%$, respectivamente. A espécie $A$. fulica manteve-se mais enterrada que $M$. oblongus $(H=50,85$; $\mathrm{p}<0,01)$, no entanto $M$. oblongus alimentou-se mais $(H=57,44 ; p<0,01)$ e explorou mais o ambiente $(H=25,39 ; p=0,02)$. Já os atos "repou-
$\operatorname{sar}^{\prime}(H=2,50 ; p=11,36)$, "deslocar" $(H=0,01$; $p=0,92)$, "enterrar" $(H=0,01 ; p=0,93)$, "defecar" $(H=0,68 ; p=0,41)$, "emergir" $(H=0,11 ; p=0,74)$ e "interagir" $(H=0,61 ; p=0,44)$ não diferiram entre as duas espécies. A frequência de "deslocar" foi relativamente baixa nas duas espécies $(15,2 \%$ para $A$. fulica e $18,1 \%$ para $M$. oblongus), porém foi observada uma tendência para descansar perto da comida, o que dispensaria a necessidade de locomoção em busca de alimento (Fig. 2).

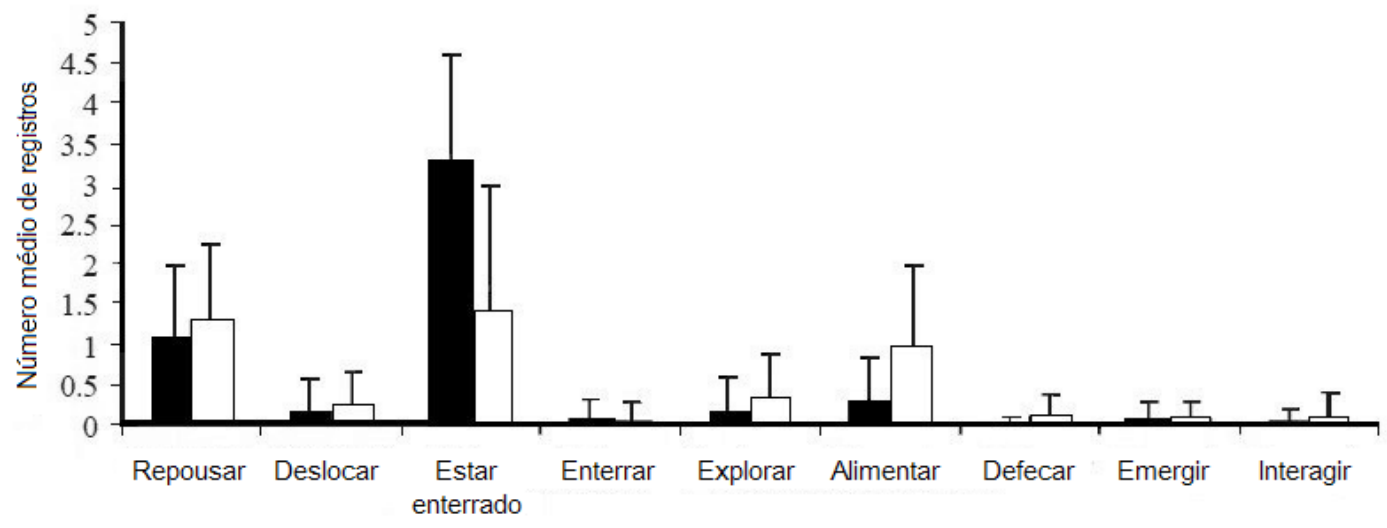

Achatina fulica $\quad \square$ Megalobulimus oblongus

Fig. 2. Número médio de registros dos atos comportamentais exibidos por Achatina fulica Bowdich, 1822 e Megalobulimus oblongus (Müller, 1774), durante a escotofase.

A média dos registros de temperatura máxima e de umidade relativa do ar durante as 24 horas consecutivas de observação em laboratório foi de $24,4^{\circ} \mathrm{C}$ e $86,2 \%$, respectivamente. A temperatura influenciou negativamente a atividade de $A$. fulica ( $r s=-0,79 ; n=24 ; p<0,01)$ e $M$. oblongus $(r s=-0,40 ; n=24 ; p<0,05)$. Já em relação à umidade relativa do ar, apenas $A$. fulica foi sensível a variações, sendo verificada uma correlação positiva ( $r s=0,50 ; n=24 ; p<0,01)$, o que não ocorreu com $M$. oblongus ( $r s=0,22 ; n=24 ; p=0,30)$.

\section{DIscussão}

A atividade noturna verificada para $A$. fulica e M. oblongus corrobora os resultados observados por outros autores para outros gastrópodes terrestres: Leptinaria unilamellata (d'Orbigny, 1835), Subulina octona (Brugüière, 1792) e Rumina decollata (Linnaeus, 1758) (Subulinidae) (Chicarino et al.; 2013; Souza et al., 2013), Bradybaena similaris (Férussac, 1821) (Xanthonychidae) (Junqueira et al., 2003), Achatina achatina (Linnaeus, 1758) (Achatinidae) (Hodasi, 1979), Deroceras reticulatum (Müller, 1774) (Agriolimacidae) (Rollo, 1991), Laevicaulis alte (Férussac, 1822) e Sarasinula linguaeformis Semper, 1885
(Veronicellidae) (Junqueira et al., 2004; Panigrahi, Mahete \& Raut, 1992; Raut \& Panigrahi, 1990), Arion intermedius Normand, 1852 e Arion lusitanicus Mabille, 1868 (Arionidae) (Bohan et al., 2000; Grimm \& Paill, 2001), Thaumastus taunaisii (Férussac, 1821) e Bulimulus tenuissimus (d'Orbigny, 1835) (Bulimulidae) (Jurberg et al., 1988; Silva et al., 2011). Como ambas as espécies dividem os mesmos locais de ocorrência (Eston et al., 2006; Santos et al., 2010) e apresentam o mesmo período de atividade, conforme observado neste estudo, provavelmente elas competem diretamente por recursos e compartiIham o mesmo nicho ecológico.

Caramujos terrestres são suscetíveis à dessecação (Cook, 2001), e o hábito noturno reduz esse risco, uma vez que geralmente observamos temperaturas mais baixas e maior umidade relativa do ar à noite. Além disso, podem evitar esse risco com o enterramento no solo, pois esse sítio de repouso protege os animais (Giokas, Pafilis \& Valakos, 2005; Hyman, 1967; Lazaridou-Dimitriadou \& Daguzan, 1981), o que confere com o presente trabalho, visto que o enterramento e o repouso no substrato foram os comportamentos mais observados em A. fulica e M. oblongus. Também não é incomum que esses comportamentos 
de moluscos terrestres ocorram em pedras e na vegetação (Hyman, 1967).

$A$ atividade locomotora conduz o animal a diferentes recursos e permite, através do muco, a liberação da água em excesso no corpo (Cook, 2001). Entretanto, esse comportamento não foi tão representativo para ambas as espécies, possivelmente pela preferência em repousar perto do alimento, observada em $A$. fulica e $M$. oblongus, o que poderia reduzir a necessidade de avançar no sentido desse recurso. Embora $A$. fulica possa se reproduzir várias vezes por ano (Eston et al., 2006), não foram observados comportamentos com fins reprodutivos nesta espécie, assim como em $M$. oblongus, o que pode estar relacionado com as baixas frequências de deslocamento e interação entre os indivíduos em ambas as espécies, e com a reduzida fecundidade de $M$. oblongus (Eston et al., 2006).

Tanto a locomoção quanto a alimentação sofrem efeito da umidade do substrato, como foi verificado por Raut \& Panigrahi (1990) para Laevicaulis alte e por Rollo (1991) para Deroceras reticulatum, sendo que este autor observou que, em condições de baixa umidade, moluscos da espécie $D$. reticulatum alimentavam-se rapidamente e retornavam aos sítios de repouso, enquanto em melhores condições de umidade deslocavam-se e alimentavam-se lentamente, utilizando o comportamento exploratório com mais frequência.

Os pulmonados terrestres podem utilizar o comportamento de exploração para perceber o ambiente e buscar recursos, já que têm quimiorrecepção bem desenvolvida com células neurossensoriais na superfície corporal relacionadas à percepção ambiental, à alimentação, à comunicação reprodutiva e à agregação (Chase \& Tolloczko, 1985; Chevalier et al., 2000; Cook, 1992; Dundee, Tizzard \& Traub, 1975; Hyman, 1967; Iglesias \& Castillejo, 1999; Stephenson, 1979). Stephenson (1979) verificou que os tentáculos de Derceras reticulatum provavelmente atuam na detecção de compostos químicos voláteis pelo olfato e não voláteis pelo paladar.

A temperatura e a umidade podem influenciar o comportamento dos moluscos terrestres, principalmente a locomoção, a alimentação, o período de atividade, a preferência de hábitat e a reprodução (Dimitrieva, 1975; Elwell \& Ulmer, 1971; Pieri \& Juberg, 1981). Segundo Cook (2001) e Kimura \& Chiba (2010), fatores abióticos são capazes de modular comunidades inteiras desses animais, principalmente a umidade, cujos efeitos na fisiologia e no comportamento relacionam-se ao equilíbrio hídrico. De modo a combater seus efeitos nocivos, tendem a exibir estratégias fisiológicas e comportamentais que reduzam perdas d'água (D'ávila et al., 2004; D'ávila \& Bessa, 2005; Giokas, Pafilis \& Valakos, 2005; Storey, 2002; Udaka et al., 2007). A espécie A. fulica é resistente a fatores abióticos (Fisher \& Colley, 2005), vivendo em uma ampla faixa de temperatura e resistindo a temperaturas extremas, fa- vorecendo, assim, a ocupação de diferentes hábitats naturais ou ambientes alterados pelo homem (Raut \& Barker, 2002). No entanto, os resultados do presente trabalho mostraram que $A$. fulica foi suscetível a variações de temperatura e umidade relativa do ar.

De modo geral, $A$. fulica foi mais ativa que a espécie nativa estudada e autores relatam os riscos de extinção de espécies nativas pela introdução de $A$. fulica, principalmente em áreas como a Mata Atlântica (Fisher \& Colley, 2005), onde $M$. oblongus também é encontrado. Apesar de $M$. oblongus ter se alimentado e explorado mais que A. fulica durante o período de observação, as características biológicas e ecológicas de $A$. fulica, como a ausência de predadores naturais, fazem dessa espécie uma competidora mais eficiente (Eston et al., 2006), capaz de colocar as populações de $M$. oblongus em risco de extinção (Colley \& Fisher, 2009; Eston et al., 2006). Além disso, A. fulica tem alto potencial reprodutivo (Colley \& Fisher, 2009; Eston et al., 2006), o que indica que a absorção de nutrientes é mais eficaz. De acordo com Byers (2000), a eficiência na conversão de recursos deve ser tão importante como a capacidade de competitividade entre essas espécies.

As populações do gênero Megalobulimus Miller, 1878 apresentam baixa densidade, crescimento lento, fecundidade reduzida e hábitos alimentares especializados, o que torna a sua condição pior quando em contato com espécies invasoras (Eston et al., 2006). Assim, apesar dos resultados obtidos para frequência de atividade de $M$. oblongus, este não pode competir em igualdade com moluscos exóticos, principalmente em reservas naturais (Fisher \& Colley, 2004, 2005).

A espécie $A$. fulica também pode atuar como um vetor de doenças endêmicas por causa de sua grande população, levando a um colapso nos moluscos de populações nativas (Raut \& Barker, 2002). As espécies invasoras causam um efeito negativo sobre a cadeia alimentar, por se tornarem um recurso excessivo de alimentos para seus predadores, levando a um aumento de suas populações que podem predar com mais intensidade não só as espécies invasoras, mas também as nativas (Colley \& Fisher, 2009).

O uso inadequado de moluscicidas químicos e o controle biológico podem causar depleção em populações do gênero Megalobulimus. Observou-se que moluscos de áreas antrópicas da família Megalobulimidae são confundidos com A. fulica, levando a um extermínio errôneo das espécies nativas (Colley \& Fisher, 2009; Fisher \& Colley, 2004). Ainda não se sabe a dimensão dos danos causados por A. fulica no Brasil, porque essa avaliação requer um estudo comparativo levando em conta as condições ambientais previamente e após a introdução (Colley \& Fisher, 2009).

O conhecimento da biologia comportamental de moluscos terrestres, de modo geral, ainda é incipiente, embora seja de grande relevância para a elaboração de estratégias de conservação 
e controle. Uma vez que existem poucos trabaIhos sobre biologia, ecologia e comportamento de espécies nativas, e vários deles são desconhecidos, torna-se difícil uma avaliação profunda do impacto a elas causado pela introdução de espécies invasoras. Assim, são necessárias outras avaliações, principalmente em campo, para estabelecer claramente a sobreposição de nichos nessas espécies.

\section{CONCLUSÃo}

De acordo com os resultados obtidos durante o presente estudo, pode-se concluir que os etogramas básicos das espécies $A$. fulica e $M$. oblongus são compostos por nove atos comportamentais, sendo dois de repouso: "repousar" e "estar enterrado", e sete de atividade: "deslocar", "enterrar", "explorar", "alimentar", "defecar", "emergir" e "interagir". Ambas as espécies apresentam horário de atividade predominantemente noturno, sendo $M$. oblongus menos ativo que $A$. fulica. A atividade de ambas as espécies se correlaciona negativamente com a temperatura e, no caso de $A$. fulica, se correlaciona positivamente com a umidade relativa do ar.

\section{Agradecimentos}

Agradecemos à mestra Paula Botelho Ferreira e às graduadas Giulia Saggioro Freesz e Tamires Moreira Vieira pela colaboração na coleta de dados, à Coordenação de Aperfeiçoamento de Pessoal de Nível Superior (CAPES) pelas bolsas concedidas e ao Instituto Federal de Educação, Ciência e Tecnologia do Sudeste de Minas Gerais (IF Sudeste MG) pelo apoio à qualificação.

\section{REFERÊNCIAS}

Altmann, J. 1974. Observational study of behaviour: sampling methods. Behaviour. 49: 227-267.

Bailey, S.E.R. 1981. Circannual and circadian rhythms in the snail Helix aspersa Müller and the photoperiodic control of annual activity and reproduction. J Comp. Physiol. 142: 89-94.

Bailey, S.E.R. 1989. Foraging behavior of terrestrial gastropods: integrating field and laboratory studies. J Mollus. Stud. 55: 263-272.

Bessa, E.C.A. \& J.L.B. Araújo. 1995a. Oviposição, tamanho de ovos e medida do comprimento da concha em diferentes fases do desenvolvimento de Subulina octona (Brugüière) (Pulmonata, Subulinidae) em condições de laboratório. Revta. Bras. Zool. 12: 647-654.
Bessa, E.C.A. \& J.L.B. Araújo. 1995b. Ocorrência de autofecundação em Subulina octona (Brugüière) (Pulmonata, Subulinidae) em condições de laboratório. Revta. Bras. Zool. 12: 719-723.

Bohan, D.A., D.M. Glen, C.W. Wiltshire \& L. Hughes. 2000. Parametric intensity and the spatial arrangement of the terrestrial herbivores Deroceras reticulatum and Arion intermedius. J Anim. Ecol. 69: 1031-1046.

Byers, J.E. 2000. Competition between two estuarine snails: implications for invasions of exotic species. Ecology. 81: 1225-1239.

Byers, J.E., S. Reichard, J.M. Randall, I.M. Parker, C.S. Smith, W.M. Londale, A.E. Atkinson, T.R. Seastedt, E. Williamson, M.E. Chornesky \& D. Hayes. 2002. Directing research to reduce the impacts of nonindigigenous species. Conserv. Biol. 16: 630-640.

Chase, R. \& B. Tolloczko. 1985. Secretory glands of the snail tentacle and their relation to the olfactory organ (Mollusca, Gastropoda). Zoomorphology. 105: 60-67.

Chase, R., R.P. Croll \& L.L. Zeichner. 1980. Aggregation in snails, Achatina fulica. Behav. Neural. Biol. 30: 218-230.

Chatfield, J.E. 1976. Studies on food and feeding in some european land molluscs. J. Conchol. 29: 5-20.

Chevalier, L., C. Desbuquois, J. Papineau \& M. Charrier. 2000. Influence of the quinolizidine alkaloid content of Lupinus albus (Fabaceae) on the feeding choice of Helix aspersa (Gastropoda: Pulmonata). J. Mollus. Stud. 66: 61-68.

Chicarino, E.D., F.A.M. Souza, V.J. Pilate, L.C. Ribeiro, E.C.A. Bessa \& L.C. Silva. 2013. Estudo comportamental comparado entre Subulina octona (Brugüière, 1789) e Leptinaria unilamellata (d'Orbigny, 1835) (Mollusca: Subulinidae). Rev. Bras. Zooc. 15: 97-105.

Colley, E. \& M.L. Fisher. 2009. Avaliação dos problemas enfrentados no manejo do caramujo gigante africano Achatina fulica (Gastropoda: Pulmonata) no Brasil. Revta. Bras. Zool. 26: 674-683.

Cook, A. 1985. Functional aspects of trail following by the carnivorous snail Euglandina rosea. Malacologia. 26: 173-181. 
Cook, A. 1992. The function of trail following in the pulmonate slug, Limax pseudoflavus. Animal Behaviour. 43: 813-821.

Cook, A. 2001. Behavioral ecology: on doing the right thing, in the right place at the right time. In: G.M. Barker (ed.). The biology of terrestrial molluscs. CABI Publishing, New York, 558p.

Coradin, L. \& D.T. Tortato. 2006. Espécies exóticas invasoras: situação brasileira. Secretaria de Biodiversidade e Florestas, MMA Brasília, 24p.

D'ávila, S. \& E.C.A. Bessa. 2005. Influência de diferentes substratos e umidade sobre o crescimento e número de ovos produzidos por Subulina octona (Brugüière) (Mollusca, Subulinidae), sob condições de laboratório. Revta. Bras. Zool. 22: 349-353.

D'ávila, S., R.J.P. Dias \& E.C.A. Bessa. 2006. Comportamento agregativo em Subulina octona (Brugüière) (Mollusca, Subulinidae). Revta. Bras. Zool. 23: 357-363.

D'ávila, S., R.J.P. Dias, E.C.A. Bessa \& E. Daemon. 2004. Resistência à dessecação em três espécies de moluscos terrestres: aspectos adaptativos e significado para o controle de helmintos. Rev. Bras. Zooc. 6: 115-127.

Dimitrieva, E.F. 1975 . The influence of temperature and moisture of the upper soil layer on the hatching intensity of the slug Deroceras reticulatum Müller. Malacol. Rev. 10: 121-132.

Dundee, D.S., M. Tizzard \& M. Traub. 1975. Aggregative behaviour in veronicellid slugs. Nautilus. 89: 69-71.

Elwell, A.S. \& M.J. Ulmer. 1971. Notes on the biology of Anguispira alternata (Stylommatophora: Endodontidae). Malacologia. 11: 199-216.

Emberton, K.C. 1994. Morphology and aestivation behaviour in some madagascan acavid land snails. Biol. J Linn. Soc. 53: 175-187.

Eston, M.R., G.V. Menezes, A.Z. Antunes, A.S.R. Santos \& A.M.R. Santos. 2006. Espécie invasora em unidade de conservação: Achatina fulica (Bowdich, 1822) no Parque Estadual Carlos Botelho, Sete Barras, SP, Brasil. Rev. Inst. Florest. 18: 173-179.
Fischer, M.L. \& E. Colley. 2004. Diagnóstico da ocorrência do caramujo gigante africano Achatina fulica Bowdich, 1822 na APA de Guaraqueçaba. Estud. Biol. 26: 3-50.

Fisher, M.L. \& E. Colley. 2005. Espécies invasoras em reservas naturais: caracterização da população de Achatina fulica Bowdich, 1822 (Mollusca - Achatinidae) na Ilha Rasa, Guaraqueçaba, Paraná, Brasil. Biota. Neotrop. 5: 127-144

Giokas, S., P. Pafilis \& E. Valakos. 2005. Ecological and physiological adaptations of the land snail Albinaria caerulea (Pulmonata, Clausilliidae). J Mollus. Stud. 71: 15-23.

Grimm, B. \& K. Schaumberger. 2002. Daily activity of the pest slug Arion lusitanicus under laboratory conditions. Ann. Appl. Biol. 141: 35-44.

Grimm, B. \& W. Paill. 2001. Spatial distribution and home-range of the pest slug Arion Iusitanicus (Mollusca: Pulmonata). Acta Oecol. 22: 219-227.

Hodasi, J.K.M. 1979. Life history studies of Achatina (Achatina) achatina (Linné). J Mollus. Stud. 45: 328-339.

Hodasi, J.K.M. 1982. The effects of different light regimes on the behavior and biology of Achatina (Achatina) achatina (Linné). J Mollus. Stud. 48: 283-293.

Hyman, L.H. 1967. The invertebrates: Mollusca I. McGraw-Hill Book Company, New York, 792p.

Iglesias, J. \& J. Castillejo. 1999. Field observations on feeding of the land snail Helix aspersa Müller. J Mollus. Stud. 65: 411-423.

IUCN - The International Union for Conservation of Nature. 2016. View 100 of the world's worst invasive alien species. Disponível em: <http://www.issg.org/worst100_ species.html>. Acesso em 18.09.2016.

Junqueira, F.O., F. Prezoto, E.C.A. Bessa, \& S. D'ávila. 2004. Horário de atividade e etograma básico de Sarasinula linguaeformis Semper, 1885 (Mollusca, Veronicellidae), em condições de laboratório. Rev. Bras. Zooc. 6: 237-247.

Junqueira, F.O., S. D'ávila, E.C.A. Bessa \& F. Prezoto. 2003. Ritmo de atividade de Bradybaena similaris (Férussac, 1821) (Mollusca, Xanthonychidae) de acordo com a idade. Rev. Etol. 5: 1-6. 
Jurberg, P., H.M. Barros, L.A.L. Gomes \& A.C.S. Coelho. 1988. Superfamília Bulimuloidea do Brasil. Bulimulidae: Thaumastus (Thaumastus) taunaisii (Férussac, 1822) com dados biológicos e aspectos comportamentais (Mollusca, Gastropoda, Pulmonata). Bol. Mus. Nac. 358: 1-47.

Kimura, K. \& S. Chiba. 2010. Interspecific interference competition alters habitat use patterns in two species of land snails. Evol. Ecol. 24: 815-825.

Kleewein, D. 1999. Population size, density, spatial distribution and dispersal in an austrian population of the land snail Arianta arbustorum styriaca (Gastropoda: Helicidae). J. Mollus. Stud. 65: 303-315.

Lazaridou-Dimitriadou, M. \& J. Daguzan. 1981. Etude de l'effet du "groupment" des individus chez Theba pisana (Mollusque Gasteropode Pulmone Stylommatophore). Malacologia. 20: 195-204.

Leahy, W.M. 1983. Comportamento e características anatomofuncionais da reprodução em Bradybaena similaris (molusco pulmonado). Cienc. Cult. 36: 1389-1392.

Pakarinen, E. 1992. Feeding avoidance of terrestrial gastropods to conspecific and nonspecific material. J Mollus. Stud. 58: 109120.

Panigrahi, A., S.K. Mahete \& S.K. Raut. 1992. Circadian rhythm in norepinephrine and epinephrine contents in the brain of the garden slug, Laevicaulis alte (Férussac). Apex. 7: 59-65.

Pieri, O. \& P. Juberg. 1981. Aspectos etológicos na sobrevivência dos caramujos vetores da xistosomose ao tratamento com moluscicidas. Mem. Inst. Oswaldo. Cruz. 76: 47-55.

Pilate, V.J., E.D. Chicarino, P.A. Daniel, E.O. Santos, L.C. Silva \& E.C.A. Bessa. 2013a. Estudo comportamental da agregação e preferência por sítio de repouso de moluscos subulinídeos em laboratório. Rev. Nord. Zool. 7: 16-26.

Pilate, V.J., L.C. Silva, T. Vargas, B.A. Souza, E.D. Chicarino \& E.C.A. Bessa. 2012. Repertório comportamental e horário de atividade do molusco terrestre Dysopeas muibum Marcus \& Marcus, 1968 (Mollusca, Subulinidae) em laboratório. BioFar: Rev. Biol. Farm. 8: 176-188.
Pilate, V.J., T. Vargas, L.C. Silva, B.A. Souza, E.D. Chicarino, E.C.A. Bessa \& B.M. Sousa. 2015. Estudo do período de atividade e dos comportamentos exibidos pelo gastrópode Zonitoides arboreus (Say, 1816) (Pulmonata, Zonitidae) em laboratório. Rev. Nord. Zool. 9: 33-46.

Pilate, V.J., T. Vargas, L.C. Silva, F.A.M. Souza, B.A. Souza, E.D. Chicarino \& E.C.A. Bessa. 2013b. Ethogram and activity pattern of Tamayoa banghaasi (Thiele, 1927) (Pulmonata, Systrophiidae) in laboratory. BioFar: Rev. Biol. Farm. 9: 1-9.

Raut, S.K. \& A. Panigrahi. 1988. Egg-nesting in the garden slug Laevicaulis alte (Férussac) (Gastropoda, Soleolifera). Malacol. Rev. 21: 101-104.

Raut, S.K. \& A. Panigrahi. 1990. Feeding rhythm in the garden slug Laevicaulis alte (Soleolifera: Veronicellidae). Malacol. Rev. 23: 39-46.

Raut, S.K. \& G.M. Barker. 2002. Achatina fulica Bowdich and others Achatinidae pest in tropical agriculture. In: G.M. Barker (ed.). Mollusks as croup pest. CABI Publishing, London, UK, 400p.

Rollo, C.D. 1991. Endogenous and exogenous regulation of activity in Deroceras reticulatum, a weather-sensitive terrestrial slug. Malacologia. 33: 199-220.

Santos, L.C., C.L. Rodrigues, G.K.M. Nunes, A.B. Barbosa, L.E.M. Lacerda, I.C. Miyahira, T.A. Viana, J.L. Oliveira, F.C. Fonseca \& P.S.C. Silva. 2010. Estado do conhecimento da fauna de invertebrados não-marinhos da Ilha Grande (Angra dos Reis, RJ). Oecol. Aust. 14: 504-549.

Silva, L.C., L.M.O. Meireles, T. Vargas, C.M. Carvalho, E.A. Gomes, F.O. Junqueira \& E.C.A. Bessa. 2011. Comportamento agregativo, preferência por sítio de repouso e variações diurnas de atividade em Bulimulus tenuissimus (d'Orbigny, 1835) (Mollusca, Bulimulidae) no laboratório. Rev. Etol. 10: 27-33.

Souza, F.A.M., V.J. Pilate, E.D. Chicarino, L.C. Ribeiro, L.C. Silva \& E.C.A. Bessa. 2013. Comparative behavioral study between juvenile and adult Rumina decollata (Mollusca, Subulinidae). Rev. Nord. Zool. 7: 1-15. 
Stephenson, J.W. 1979. The functioning of the sense organs associated with feeding behaviour in Deroceras reticulatum (Müll.). J Mollus. Stud. 45: 167-171.

Storey, K.B. 2002. Life in the slow lane: molecular mechanisms of estivation. Comp. Biochem. Phys. Part. A. 133: 733-754.
Udaka, H., M. Mori, S.G. Goto \& H. Numata. 2007. Seasonal reproductive cycle in relation to tolerance to high temperatures in the terrestrial slug Lehmannia valentiana. Invert. Biol. 126: 154-162.

Vasconcellos, M.C. \& E. Pile. 2001. Ocorrência de Achatina fulica no Vale do Paraíba, Estado do Rio de Janeiro, Brasil. Rev. Saúde Pública. 35: 582-584.

Recebido em 18.IX.2016 Aceito em 20.VI.2017 